Check for updates

Cite this: RSC Adv., 2018, 8, 3099

Received 27th November 2017

Accepted 8th January 2018

DOI: 10.1039/c7ra12827d

rsc.li/rsc-advances

\section{Critical phenomena and estimation of the spontaneous magnetization from a mean field analysis of the magnetic entropy change in $\mathrm{La}_{0.7} \mathrm{Ca}_{0.1} \mathrm{~Pb}_{0.2} \mathrm{Mn}_{0.95} \mathrm{Al}_{0.025} \mathrm{Sn}_{0.025} \mathrm{O}_{3}$}

\begin{abstract}
Khadija Dhahri, (DD *a N. Dhahri, ${ }^{\text {a }}$ J. Dhahri, (D) ${ }^{a}$ K. Taibi ${ }^{b}$ and E. K. Hlil ${ }^{c}$
In the present work, we have studied the universal critical behavior in the perovskite-manganite compound $\mathrm{La}_{0.7} \mathrm{Ca}_{0.1} \mathrm{~Pb}_{0.2} \mathrm{Mn}_{0.95} \mathrm{Al}_{0.025} \mathrm{Sn}_{0.025} \mathrm{O}_{3}$. Experimental results revealed that all samples exhibit a second-order magnetic phase transition. To study the critical behavior of the paramagnetic-ferromagnetic transition, various techniques such as modified Arrott plots, the Kouvel-Fisher method and critical isotherm analysis were used to determine the values of the Curie temperature $T_{C}$, as well as the critical exponents $\beta$ (corresponding to the spontaneous magnetization), $\gamma$ (corresponding to the initial susceptibility) and $\delta$ (corresponding to the critical magnetization isotherm). The critical exponent values for our sample are consistent with the prediction of the mean field model $\left(\beta=0.46, \gamma=1.0\right.$, and $\delta=2.94$ at an average $T_{\mathrm{C}}=$ $302.12 \mathrm{~K}$ ), indicating that the magnetic interactions are long-range. The reliability of the critical exponent values was confirmed by the Widom scaling relation (WSR) and the universal scaling hypothesis. Moreover, to estimate the spontaneous magnetization of $\mathrm{La}_{0.7} \mathrm{Ca}_{0.1} \mathrm{~Pb}_{0.2} \mathrm{Mn}_{0.95} \mathrm{Al}_{0.025} \mathrm{Sn}_{0.025} \mathrm{O}_{3}$ perovskite, we used the magnetic entropy change $\left(\Delta S_{M}\right)$, obtained from isothermal magnetization. The results obtained through this approach are compared to those obtained from classical analysis using Arrott curves. An excellent agreement is found between this approach and the one obtained from the extrapolation of the Arrott curves.
\end{abstract}

\section{Introduction}

Over the past few years, magnetic materials have had potential applications in information storage and magnetic sensors, these materials have been also found to exhibit a large magnetocaloric effect (MCE), revealing that they can be considered as promising magnetic refrigerants. ${ }^{1,2}$ The MCE, which is an intrinsic property of magnetic materials, is defined as the thermal response (heating or cooling) of magnetic solids during the application or removal of an external magnetic field. Nowadays, the majority of research in this area is to develop suitable materials that are cost effective and exhibit large MCEs (large isothermal magnetic entropy change $\Delta S_{\mathrm{M}}$ or large adiabatic temperature change $\Delta T_{\mathrm{ad}}$ ) over a wide temperature range. These materials exhibit a rich variety of physical properties. The most accepted interpretations for the cause of these properties are the double exchange model ${ }^{3}$ and the Jahn-Teller effect., ${ }^{4,5}$ Both of these mechanisms are used to identify the magnetic phase transition (FM-PM). However, the perovskite manganites with mixed valence are intrinsically

\footnotetext{
${ }^{a}$ Laboratoire de la Matière Condensée et des Nanosciences, Département de Physique, Faculté des Sciences, Université de Monastir, Monastir 5019, Tunisia. E-mail: khadijadhahri91@yahoo.com

${ }^{b}$ Département SDM, FGMGP/USTHB, 16311, Algeria

'Institut Néel, CNRS, Université J. Fourier, BP 166, 38042 Gronoble, France
}

inhomogenous, both above and below the Curie temperature. In this context, a detailed investigation of the critical exponents in this region is needed to give an idea about the order of the phase transition and the common universality class. For this object, it is required to investigate in details the critical exponents at the region of the PM-FM transition. Historically, the critical behavior in the DE model was first described by long-range mean-field theory. Sequentially, depending on the computational technology for the colossal magnetoresistance (CMR) manganites, many works suggested that the critical behavior should be attributed to short-range Heisenberg model $^{6,7}$ with only a nearest neighbor exchange coupling assuming a short-range interaction between localized spins. Based on the study of dc magnetization, Ghosh et al. ${ }^{8}$ reported that the critical exponent $\beta$ is equal to 0.37 for the ferromagnet manganite $\mathrm{La}_{0.7} \mathrm{Sr}_{0.3} \mathrm{MnO}_{3}$. Nevertheless, a relative high value of $\beta=0.5$ obtained in the polycrystalline $\mathrm{La}_{0.8} \mathrm{Sr}_{0.2} \mathrm{MnO}_{3}$ is in good agreement with that in mean-field model. ${ }^{9}$ Other critical behavior studies in manganites ${ }^{\mathbf{1 0 - 1 2}}$ have been carried out on the vicinity of the magnetic phase transition by using a variety of techniques have yielded a wide range of values for the critical parameters $\beta$ of the magnetization. The values range, from about 0.3 to 0.5 , includes the mean-field $(\beta=$ $0.5)$, the $3 \mathrm{D}$ isotropic nearest-neighbor Heisenberg $(\beta=0.365)$ and the $3 \mathrm{D}$-Ising $(\beta=0.325)$ estimates and those that cannot be classified into any universality class ever known. ${ }^{13}$ 
In this paper, we propose to present a detailed analysis of the critical phenomena in the perovskite. We demonstrate that the values of critical exponents $\beta, \gamma$, and $\delta$ of our sample are close to the theoretical prediction of the mean-field model indicating the existence of a long-range ferromagnetic interaction. Moreover, we utilize the isothermal magnetic entropy change $\left(-\Delta S_{\mathrm{M}}\right)$ obtained from isothermal magnetization measurements, in mean-field model, to estimate the spontaneous magnetization for our system and then compared to results obtained from Arrott curves. ${ }^{14}$

\section{Experimental details}

$\mathrm{La}_{0.7} \mathrm{Ca}_{0.1} \mathrm{~Pb}_{0.2} \mathrm{Mn}_{0.95} \mathrm{Al}_{0.025} \mathrm{Sn}_{0.025} \mathrm{O}_{3}$ polycrystalline has been synthesized by sol-gel method that details was described in our previous work. ${ }^{15}$ The variations of the magnetization as a function of magnetic field at different temperatures have been measured between 0 and $5 \mathrm{~T}$ and in a temperature range around the $T_{\mathrm{C}}$ using BS1 and BS2 magnetometers developed in Louis Neel Laboratory at Grenoble. To extract the critical exponents of the sample accurately, the magnetic isotherms were measured in the range of $0-5 \mathrm{~T}$ and with a temperature interval of $3 \mathrm{~K}$ in the vicinity of its Curie temperatures $\left(T_{\mathrm{C}}\right)$.

\section{Results and discussion}

\subsection{Magnetic and magnetocaloric effect properties}

Fig. 1 displays the zero field cooled (ZFC) and field-cooled (FC) magnetization evolution versus temperature taken at an applied field of $\mu_{0} H=0.05 \mathrm{~T}$ for $\mathrm{La}_{0.7} \mathrm{Ca}_{0.1} \mathrm{~Pb}_{0.2} \mathrm{Mn}_{0.95} \mathrm{Al}_{0.025} \mathrm{Sn}_{0.025} \mathrm{O}_{3}$ compound. It is observed from Fig. 1 that the $M(T)$ curve exhibits a single magnetic transition from paramagnetic (PM) to ferromagnetic (FM) behavior as the temperature decreases. The transition temperature $T_{\mathrm{C}}$ for our sample (inset Fig. 1), determined from the critical point in the derivative $\mathrm{d} M / \mathrm{d} T$ curves, is found to be $300 \mathrm{~K}^{15}$ This deduced value is equal to room temperature, which is quite suitable for magnetic refrigeration

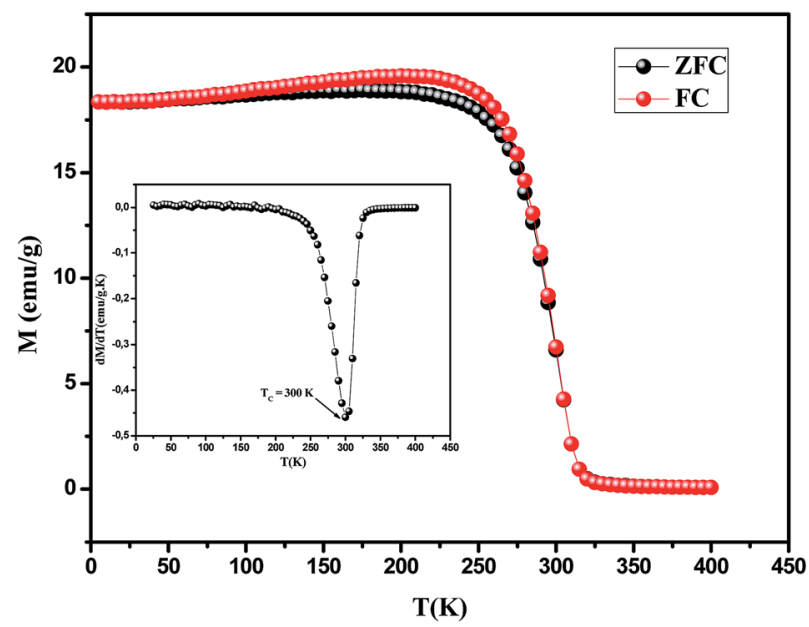

Fig. 1 ZFC and FC magnetization as a function of temperature and inset shows the corresponding $\mathrm{d} M / \mathrm{d} T$ versus $T$. technology. Furthermore, we have also notice from $M-T$ curves that the ZFC and FC curves coincide above $T_{\mathrm{C}}$ as expected in the paramagnetic state, while they slightly split below $T_{\mathrm{C}}$ suggesting the existence of a multi-domain magnetic state. ${ }^{16,17}$

Following the magnetic properties, we measured magneticfield dependences of the initial magnetization, $M(H)$, for $\mathrm{La}_{0.7^{-}}$ $\mathrm{Ca}_{0.1} \mathrm{~Pb}_{0.2} \mathrm{Mn}_{0.95} \mathrm{Al}_{0.025} \mathrm{Sn}_{0.025} \mathrm{O}_{3}$ sample around their $\mathrm{FM}-\mathrm{PM}$ phase transition. Fig. 2 represents the magnetic field dependence of magnetization at various temperatures between 240 and $342 \mathrm{~K}$ under magnetic field $\mu_{0} H$ ranging between 1 and $5 \mathrm{~T}$. Characteristic $M(H)$ curves of manganites usually exhibit a very high increase in $M$ at low fields and then a gradual saturation at high fields, as expected for a ferromagnetic material. The saturated magnetization $\left(M_{\mathrm{S}}\right)$ can be estimated from an extrapolation of the high field $M-\mu_{0} H$ curve to $\mu_{0} H=0$, and the obtained $M_{\mathrm{S}}$ is $44 \mathrm{emu} \mathrm{g}^{-1}$ at $T=240 \mathrm{~K}$. According to the Maxwell relations based on the thermodynamical theory, the magnetic entropy change $\left(\left|\Delta S_{\mathrm{M}}\right|\right)$, an important thermodynamic parameter characteristic of the disorder of magnetic moments can be evaluated from the temperature and field dependent magnetization curves, using a numerical approximation as follows:

$$
\Delta S_{\mathrm{M}}(T, H)=S_{\mathrm{M}}(T, H)-S_{\mathrm{M}}(T, 0)=\int_{0}^{H}\left(\frac{\mathrm{d} M}{\mathrm{~d} T}\right)_{H} \mathrm{~d} H
$$

The magnetic entropy change between 0 and $\mu_{0} H$ magnetic applied field, can be basically obtained by:

$$
\begin{aligned}
\Delta S_{\mathrm{M}}\left(\frac{T_{1}+T_{2}}{2}\right)= & \frac{1}{T_{2}-T_{1}}\left[\int_{0}^{\mu_{0} H} M\left(T_{2}, \mu_{0} H\right) \mathrm{d} \mu_{0} H\right. \\
& \left.-\int_{0}^{\mu_{0} H} M\left(T_{1}, \mu_{0} H\right) \mathrm{d} \mu_{0} H\right]
\end{aligned}
$$

The obtained magnetic entropy change has been plotted as a function of temperature in the inset of Fig. 2. One can notice

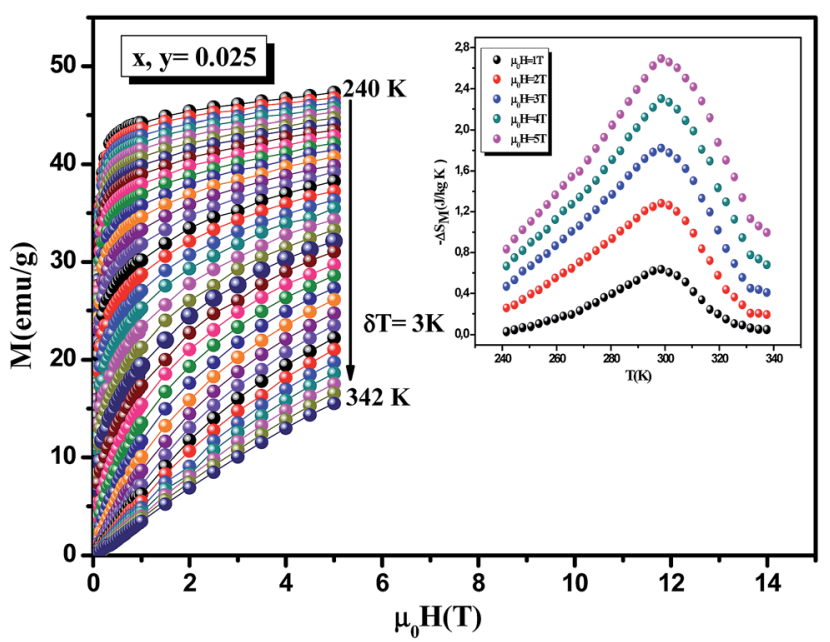

Fig. 2 Magnetic field dependence of magnetization at several temperatures for $\mathrm{La}_{0.7} \mathrm{Ca}_{0.1} \mathrm{~Pb}_{0.2} \mathrm{Mn}_{0.95} \mathrm{Al}_{0.025} \mathrm{Sn}_{0.025} \mathrm{O}_{3}$ sample, the inset: temperature dependence of the magnetic entropy change $\left(-\Delta S_{M}\right)$ at different applied magnetic field change intervals. 
that the maximum in magnetic entropy change $\left(\left|\Delta S_{\mathrm{M}}^{\max }\right|\right)$ exhibits a peak around $T_{\mathrm{C}}$ and increases with increasing of applied magnetic field. The maximum value of $\Delta S_{\mathrm{M}}$ observed for the sample under an applied magnetic field of $5 \mathrm{~T}$ was found to be about $2.7 \mathrm{~J} \mathrm{~kg}^{-1} \mathrm{~K}^{-1}$, which is comparable to some typical manganites (Table 1). Besides magnetic entropy change, there is another important parameter that measures the cooling efficiency of the refrigerant is the relative cooling power (RCP), which can be evaluated by considering the magnitude of $\left|\Delta S_{\mathrm{M}}^{\max }\right|$ and its full width at half maximum $\left(\delta T_{\mathrm{FWHM}}\right)$ and it corresponds to the amount of heat transferred between the cold and hot sides in an ideal refrigeration cycle. The obtained value of RCP in an applied field of $5 \mathrm{~T}$ is given in Table 1. For comparison purposes, we gathered in Table 1 the values of the $\left(-\Delta S_{\mathrm{M}}^{\max }\right)$ and RCP for our sample under a magnetic applied field of $5 \mathrm{~T}$ and several others results reported in the literature. ${ }^{18-24}$ As we can see through this assessment, our material can be a leader system in the magnetic refrigeration offering the opportunity to use it in a broad temperature range.

\subsection{Critical behavior}

Generally, to further understand the nature of the ferromagnetic-paramagnetic (FM-PM) phase transition (first or second order), the Arrott plots $M^{1 / \beta} v s$. $(H / M)^{1 / \gamma}$ following mean-field theory $\beta=0.5$ and $\gamma=1$ are presented in Fig. 3. Based on the criterion proposed by Banerjee, ${ }^{25}$ the magnetic transition is of second order if all the $H / M v s . M^{2}$ curves have a positive slope while the negative slope of $M^{2} v s . H / M$ reflects that the magnetic system exhibits a first order transition. In agreement with Mira et al. ${ }^{26}$ it can be observed from these curves that the $H / M v s . M^{2}$ curves of our sample exhibit a positive slope and we analyze our data assuming the PM-FM transition to be of second order in this compound according to Banerjee's criteria. According to the mean field model, such curves should reveal a linear behavior in the high field region at different temperatures and the line at $T=T_{\mathrm{C}}$ should just pass through the origin. ${ }^{27}$ It is evident in Fig. 3 that the line of $M^{2} v s$. $H / M$ crosses the origin at $T_{\mathrm{C}}=300 \mathrm{~K}$, suggesting that the mean-field theory can describe the critical behavior of this sample.

A deeper insight into the magnetic phase transition may be obtained by analyzing the critical phenomena. Generally, at high field region, the effect of charge, lattice, and orbital

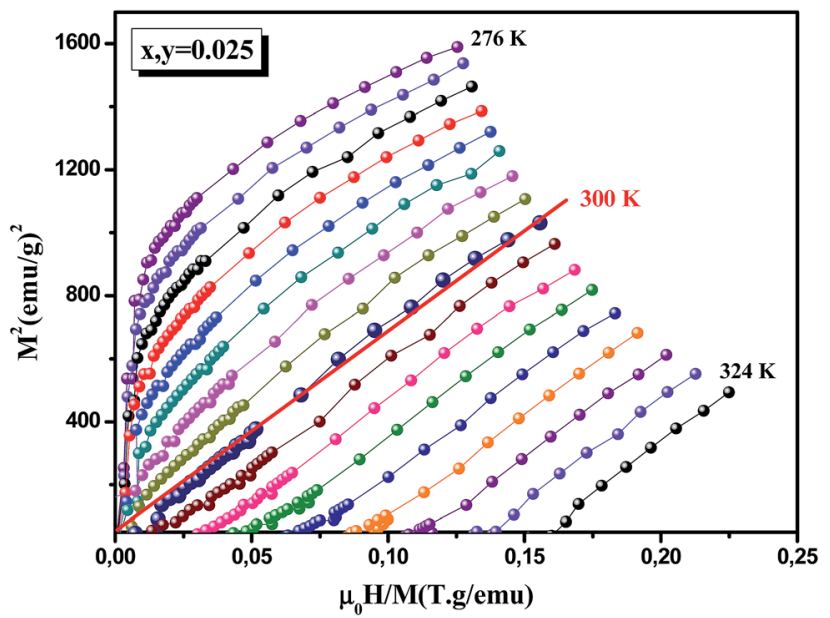

Fig. 3 Arrott plots $\left(M^{2}\right.$ versus $\left.\mu_{0} H / M\right)$ of $\mathrm{La}_{0.7} \mathrm{Ca}_{0.1} \mathrm{~Pb}_{0.2} \mathrm{Mn}_{0.95} \mathrm{Al}_{0.025^{-}}$ $\mathrm{Sn}_{0.025} \mathrm{O}_{3}$ compound at different temperatures around $T_{\mathrm{C}}$.

degrees of freedom are suppressed in a ferromagnet and the order parameter can be identified with the macroscopic magnetization. In the present case and in order to seek adequate model leading to a set of reasonably good parallel straight lines and the correct $\beta$ and $\gamma$ exponents, the data was analyzed using a modified Arrott-plot expression $\left(M^{1 / \beta} v s\right.$. $\left.(H / M)^{1 / \gamma}\right)$, based on the so-called Arrott-Noakes equation of state: ${ }^{28}$

$$
(H / M)^{1 / \gamma}=a\left(T-T_{\mathrm{C}}\right) / T+b M^{1 / \beta}
$$

where $a$ and $b$ are considered to be constants.

The MAP isotherms of $M^{1 / \beta}$ versus $(H / M)^{1 / \gamma}$ are plotted for $\mathrm{La}_{0.7} \mathrm{Ca}_{0.1} \mathrm{~Pb}_{0.2} \mathrm{Mn}_{0.95} \mathrm{Al}_{0.025} \mathrm{Sn}_{0.025} \mathrm{O}_{3}$ at different temperatures by using four models of critical exponents. Fig. 4a demonstrates the tricritical mean-field model $(\beta=0.25, \gamma=1)$, Fig. $4 \mathrm{~b}$ illustrates the 3D-Heisenberg model $(\beta=0.365, \gamma=1.336)$ and Fig. 4c shows the 3D-Ising model $(\beta=0.325, \gamma=1.24) .{ }^{27-30}$

According to the scaling hypothesis, the second-order magnetic phase transition is characterized by a set of critical exponents $\beta$ (associated with the spontaneous magnetization $M$ $(H=0)$ below $\left.T_{\mathrm{C}}\right), \gamma$ (related to the initial susceptibility $\chi_{0}{ }^{-1}$ above $T_{\mathrm{C}}$ ) and $\delta$ (related to the critical magnetization at $\left.T_{\mathrm{C}}\right)^{31,32}$ They are defined as follows:

Table 1 Maximum entropy change $\left|\Delta S_{M}^{\max }\right|$ and relative cooling power (RCP), for $\mathrm{La}_{0.7} \mathrm{Ca}_{0.1} \mathrm{~Pb}_{0.2} \mathrm{Mn}_{0.95} \mathrm{Al}_{0.025} \mathrm{Sn}_{0.025} \mathrm{O}_{3}$, occurring at the Curie temperature $\left(T_{\mathrm{C}}\right)$ and under magnetic field variations, $\Delta H=1 \mathrm{~T}, 3 \mathrm{~T}$ or $\Delta H=5 \mathrm{~T}$, compared to several materials considered for magnetic refrigeration

\begin{tabular}{|c|c|c|c|c|c|}
\hline Sample & $T_{\mathrm{C}}(\mathrm{K})$ & $\Delta H(\mathrm{~T})$ & $-\Delta S_{\mathrm{M}}^{\max }\left(\mathrm{J} \mathrm{kg}^{-1} \mathrm{~K}^{-1}\right)$ & $\operatorname{RCP}\left(\mathrm{Jkg}^{-1}\right)$ & References \\
\hline $\mathrm{Gd}$ & 293 & 5 & 9.5 & 410 & 18 \\
\hline $\mathrm{Gd}_{5} \mathrm{Si}_{2} \mathrm{Ge}_{2}$ & 275 & 5 & 18.5 & 535 & 19 \\
\hline $\mathrm{La}_{0.67} \mathrm{Ba}_{0.33} \mathrm{MnO}_{3}$ & 292 & 5 & 1.48 & 161 & 20 \\
\hline $\mathrm{La}_{0.7} \mathrm{Sr}_{0.3} \mathrm{Mn}_{0.9} \mathrm{Al}_{0.1} \mathrm{O}_{3}$ & 310 & 5 & 2.6 & 109 & 21 \\
\hline $\mathrm{La}_{0.67} \mathrm{Ca}_{0.33} \mathrm{MnO}_{3}$ & 252 & 5 & 2.06 & 175 & 19 \\
\hline $\mathrm{La}_{2 / 3}(\mathrm{Ca}, \mathrm{Pb})_{1 / 3} \mathrm{MnO}_{3}$ & 290 & 7 & 7.5 & 375 & 22 \\
\hline $\mathrm{La}_{0.65} \mathrm{Ca}_{0.35} \mathrm{Ti}_{0.4} \mathrm{Mn}_{0.6} \mathrm{O}_{3}$ & 42 & 3 & 0.6 & 55 & 23 \\
\hline $\mathrm{La}_{0.7} \mathrm{~Pb}_{0.3} \mathrm{MnO}_{3}$ & 358 & 1.35 & 1.53 & 53 & 24 \\
\hline $\mathrm{La}_{0.7} \mathrm{Ca}_{0.1} \mathrm{~Pb}_{0.2} \mathrm{Mn}_{0.95} \mathrm{Al}_{0.025} \mathrm{Sn}_{0.025} \mathrm{O}_{3}$ & 300 & 5 & 2.7 & 137 & This work \\
\hline
\end{tabular}



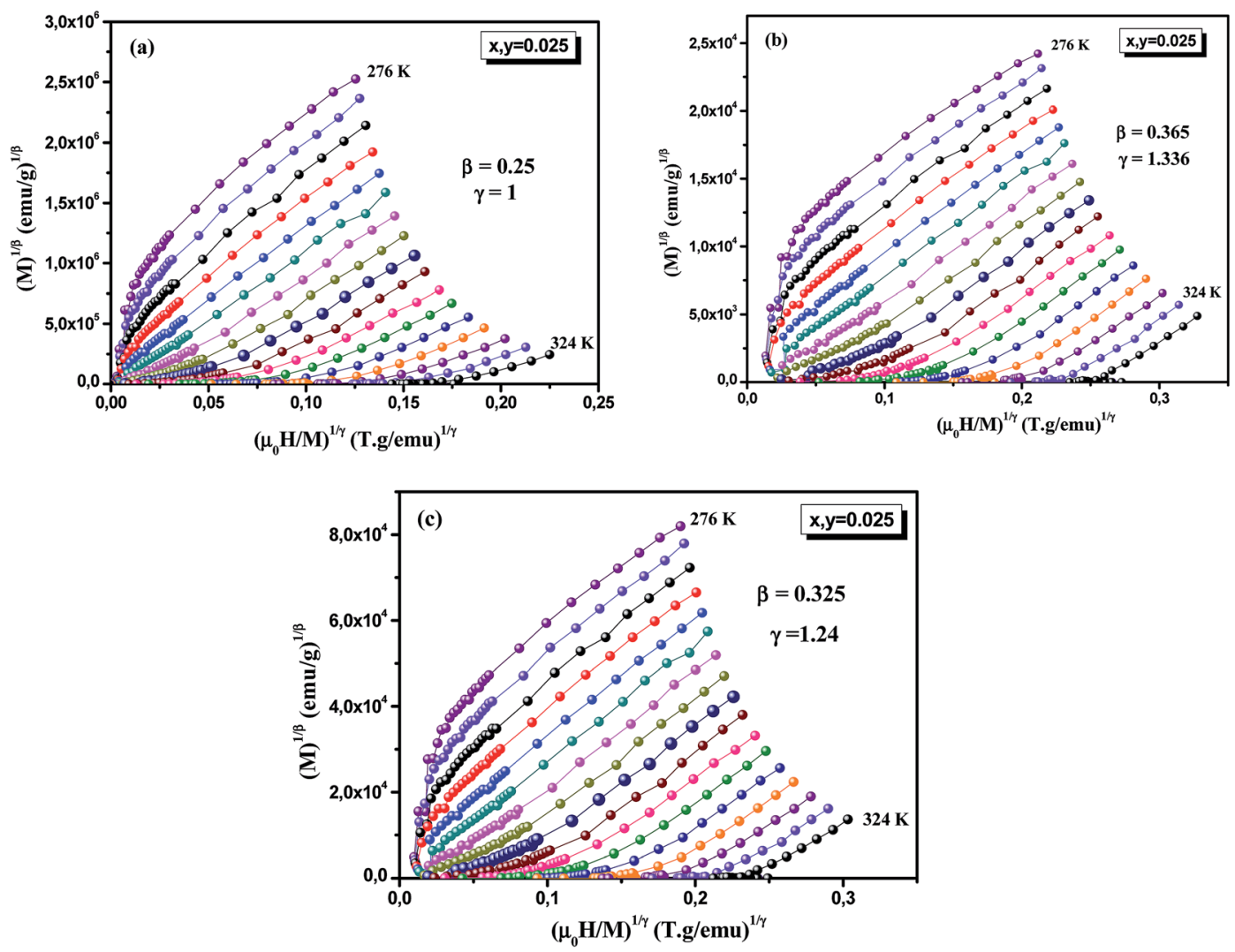

Fig. 4 Modified Arrott plots: isotherms of $M^{1 / \beta}$ vs. $\mu_{0} H / M^{1 / \gamma}$ with (a) tricritical mean-field model, (b) 3D-Heisenberg model, (c) 3D-Ising model.

$$
\begin{gathered}
M_{\mathrm{S}}(T)=M_{0}(-\varepsilon)^{\beta}, \varepsilon<0 \\
\chi_{0}^{-1}(T)=\left(h_{0} / M_{0}\right) \varepsilon^{\gamma}, \varepsilon>0 \\
M=D H^{1 / \delta}, \varepsilon=0
\end{gathered}
$$

where $\varepsilon$ is the reduced temperature $\varepsilon=T-T_{\mathrm{C}} / T_{\mathrm{C}}$, and $M_{0}$, $h_{0} / M_{0}$, and $D$ are the critical amplitudes.

Following a standard procedure, we could calculate the values of the spontaneous magnetization $M_{\mathrm{S}}(T)$ as well as the inverse susceptibility $\chi_{0}{ }^{-1}(T)$ from a linear extrapolation in the high fields to the intercepts with the axes $M^{1 / \beta}$ and $(H / M)^{1 / \gamma}$, respectively. The $M_{\mathrm{S}}(T)$ and $\chi_{0}{ }^{-1}(T)$ are displayed in Fig. 5. By fitting the $M_{\mathrm{S}}(T)$ and $\chi_{0}{ }^{-1}(T)$ data to eqn (4) and (5), respectively, new values of $\beta, \gamma$ and $T_{\mathrm{C}}$ will be achieved and listed in Table 2 .

In addition, for comparison with the critical exponents previously calculated, these exponents can also be determined more accurately according to the Kouvel-Fisher (KF) method. This method is an alternative analytical expression of the power law defined as: ${ }^{33}$

$$
\begin{gathered}
\frac{M_{\mathrm{S}}(T)}{\mathrm{d} M_{\mathrm{S}}(T) / \mathrm{d} T}=\frac{T-T_{\mathrm{C}}}{\beta} \\
\frac{\chi_{0}^{-1}(T)}{\mathrm{d} \chi_{0}^{-1}(T) / \mathrm{d} T}=\frac{T-T_{\mathrm{C}}}{\gamma}
\end{gathered}
$$

According to this later equation, the plots of $M_{\mathrm{S}}(T)\left(\mathrm{d} M_{\mathrm{S}}(T) /\right.$ $\mathrm{d} T)^{-1}$ vs. $T$ and $\chi_{0}{ }^{-1}(T)\left(\mathrm{d} \chi_{0}{ }^{-1}(T) / \mathrm{d} T\right)^{-1}$ vs. $T$ (Fig. 6) yield straight lines with slopes $1 / \beta$ and $1 / \gamma$, respectively and the intercepts on $T$ axes are equal to Curie temperature $\left(T_{\mathrm{C}}\right)$. From the fitted straight line in KF plots, we have obtained the critical exponents which are in agreement with those deduced from the previous calculations in allowable error. Besides, the final parameter delta ' $\delta$ ' can be determined directly from the critical isotherm

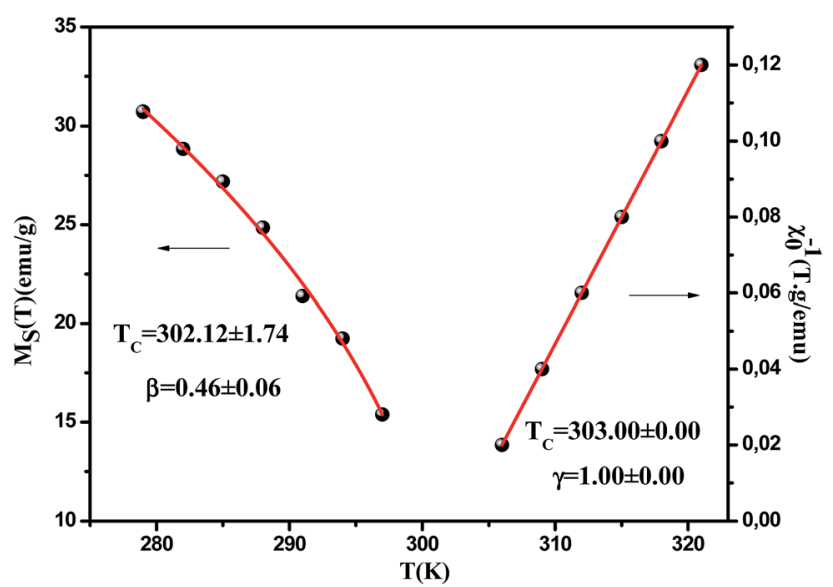

Fig. 5 The spontaneous magnetization $M_{S}(T)$ and the inverse initial susceptibility $\chi_{0}{ }^{-1}(T)$ along with the fitting curves. 
Table 2 Comparison of critical exponents of $\mathrm{La}_{0.7} \mathrm{Ca}_{0.1} \mathrm{~Pb}_{0.2} \mathrm{Mn}_{0.95} \mathrm{Al}_{0.025} \mathrm{Sn}_{0.025} \mathrm{O}_{3}$ with other reports and various theoretical models

\begin{tabular}{|c|c|c|c|c|c|c|}
\hline Samples & Techniques & $T_{\mathrm{C}}(\mathrm{K})$ & $\beta$ & $\gamma$ & $\delta$ & Ref. \\
\hline \multirow[t]{2}{*}{$\mathrm{La}_{0.7} \mathrm{Ca}_{0.1} \mathrm{~Pb}_{0.2} \mathrm{Mn}_{0.85} \mathrm{Al}_{0.075} \mathrm{Sn}_{0.075} \mathrm{O}_{3}$} & MAP & $302.12 \pm 1.74$ & $0.46 \pm 0.06$ & $1.00 \pm 0.00$ & \multirow[t]{2}{*}{2.94} & \multirow[t]{2}{*}{ This work } \\
\hline & KF & $301.86 \pm 0.0$ & $0.44 \pm 0.04$ & $1.0 \pm 0.0$ & & \\
\hline 3D-Ising mode & Theory & - & 0.325 & 1.24 & 4.82 & 43 \\
\hline Tricritical mean field model & Theory & - & 0.25 & 1 & 5 & 44 \\
\hline 3D-Heseinberg model & Theory & - & 0.365 & 1.336 & 4.8 & 45 \\
\hline $\mathrm{La}_{0.67} \mathrm{~Pb}_{0.33} \mathrm{MnO}_{3}$ & & 360.41 & 0.367 & 1.22 & 4.29 & 35 \\
\hline $\mathrm{LaMn}_{0.9} \mathrm{Ti}_{0.1} \mathrm{O}_{3}$ & & $145.3 \pm 0.1$ & $0.375 \pm 0.00$ & $1.25 \pm 0.02$ & 4.11 & 39 \\
\hline $\mathrm{Nd}_{0.7} \mathrm{~Pb}_{0.3} \mathrm{MnO}_{3}$ & & 148 & $0.361 \pm 0.001$ & $1.325 \pm 0.001$ & 4.62 & 40 \\
\hline $\mathrm{La}_{0.7} \mathrm{Sr}_{0.3} \mathrm{Mn}_{0.98} \mathrm{Ni}_{0.02} \mathrm{O}_{3}$ & & 352.6 & $0.400 \pm 0.017$ & $1.082 \pm 0.032$ & 3.79 & 41 \\
\hline $\mathrm{La}_{0.7} \mathrm{Sr}_{0.3} \mathrm{MnO}_{3}$ & & 360 & $0.45 \pm 0.02$ & $1.08 \pm 0.04$ & 3.04 & 42 \\
\hline
\end{tabular}

$M\left(T_{\mathrm{C}}, \mu_{0} H\right)$. Fig. 7 shows the magnetic field dependence of magnetization at $T=T_{\mathrm{C}}$ for our sample and in the inset we presented the same plot in log-log scale. According to eqn (6), $\log (M)$ vs. $\log (H)$ plot would give a straight line with slope $1 / \delta$, $\delta$ is yielded as 2.94 .

Another way to estimate the critical component $\delta$, from $\beta$ and $\gamma$, is possible by using the Widom scaling relation shown in eqn (9): $:^{31,34}$

$$
\delta=1+\frac{\gamma}{\beta}
$$

Using this relation and the obtained values of $\beta$ and $\gamma$ from the mean-field method, we obtain $\delta=3.2$. One can see that the parameter $\delta$ obtained from the scaling relation is relatively close to the estimated $\delta=2.94$ value from the critical isotherms at $T_{\mathrm{C}}$ $=300 \mathrm{~K}$. As consequence, Widom scaling relation is a good tool for checking the reasonability of the critical exponents obtained from the experimental data. The Widom relationship has been tested by plotting $M\left(T_{\mathrm{C}}\right)$ versus $\left(\mu_{0} H\right)^{\beta /(\beta+\gamma)}$ and checking the linearity of the curve as shown in Fig. 8.

The reliability of the obtained values could be tested according to the prediction of the scaling hypothesis, which

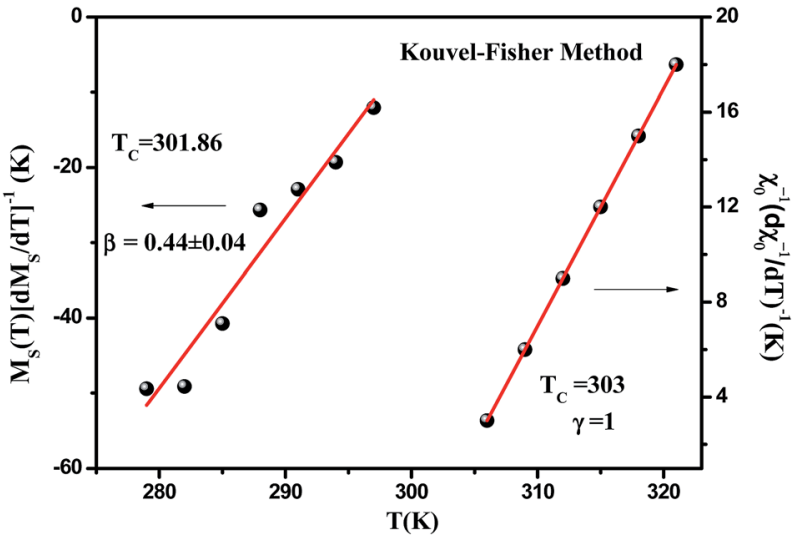

Fig. 6 Kouvel-Fisher plots for $\mathrm{La}_{0.7} \mathrm{Ca}_{0.1} \mathrm{~Pb}_{0.2} \mathrm{Mn}_{0.95} \mathrm{Al}_{0.025} \mathrm{Sn}_{0.025} \mathrm{O}_{3}$ manganite. The solid lines are the linear fits of the symbols. suggests that in the critical region the isothermal magnetization $M$ is a function of $\varepsilon$ and $\mu_{0} H:^{35,36}$

$$
M\left(\mu_{0} H, \varepsilon\right)=\varepsilon^{\beta} f_{ \pm}\left(\mu_{0} H / \varepsilon^{\beta+\gamma}\right)
$$

where $f_{+}$for $T>T_{\mathrm{C}}$ and $f_{-}$for $T<T_{\mathrm{C}}$, are regular analytical functions. Eqn (10) implies that $M / \varepsilon^{\beta} v s . H / \varepsilon^{\beta+\gamma}$ falls on two universal curves: one for temperatures $T>T_{\mathrm{C}}(\varepsilon>0)$ and the other for $T<T_{\mathrm{C}}(\varepsilon<0)$. Using the values of $\beta$ and $\gamma$ obtained by the KF method, the scaled data are plotted in Fig. 9 and the inset shows the same data in a logarithmic scale. The experimental data reveal that all points fall on two curves, one for $T<$ $T_{\mathrm{C}}$ and the other for $T>T_{\mathrm{C}}$. This proves the validity of these critical parameters and $T_{\mathrm{C}}$ temperature and the good accordance with the scaling hypothesis.

To put our obtained results in the context of previous works, we have listed the critical exponents of $\mathrm{La}_{0.7} \mathrm{Ca}_{0.1} \mathrm{~Pb}_{0.2} \mathrm{Mn}_{0.95^{-}}$ $\mathrm{Al}_{0.025} \mathrm{Sn}_{0.025} \mathrm{O}_{3}$, those of some manganites available in the literature ${ }^{35,37-42}$ and the theoretical values based on the standard models ${ }^{4-45}$ in Table 2. As mentioned above, it can be clearly

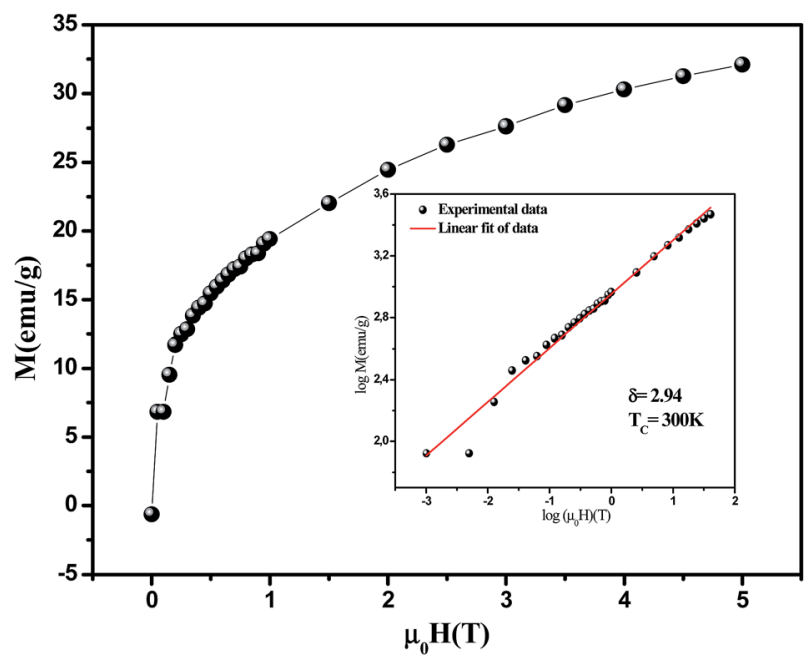

Fig. 7 Critical isotherms of $M$ vs. $\mu_{0} H$ for $\mathrm{La}_{0.7} \mathrm{Ca}_{0.1} \mathrm{~Pb}_{0.2} \mathrm{Mn}_{0.95^{-}}$ $\mathrm{Al}_{0.025} \mathrm{Sn}_{0.025} \mathrm{O}_{3}$. The inset shows the $\ln -\ln$ scale for $M$ vs. $\mu_{0} H$. 


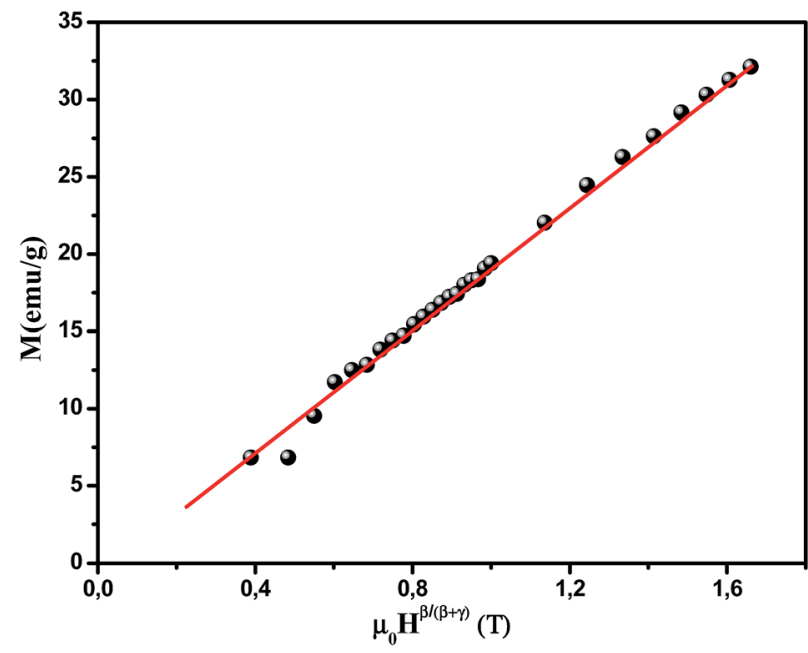

Fig. 8 The linearity of the $M\left(T=T_{C}\right)$ versus $\mu_{0} H^{\beta /(\beta+\gamma)}$ curves validates the value of $\delta$ calculated using the Widom relationship with the experimental data.

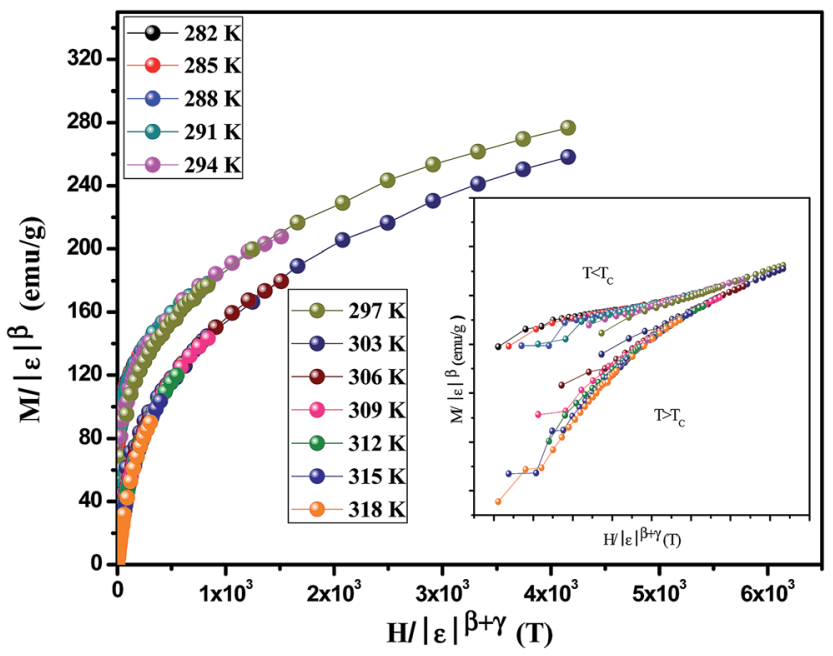

Fig. 9 Scaling plots indicating two universal curves below and above $T_{C}$. Inset shows the same plots on a log-log scale.

seen that these exponents are quite close to those expected for the mean field theory $(\beta=0.5, \gamma=1$, and $\delta=3) .{ }^{9}$ This means that there exists long-range FM order in the $\mathrm{La}_{0.7} \mathrm{Ca}_{0.1} \mathrm{~Pb}_{0.2^{-}}$ $\mathrm{Mn}_{0.95} \mathrm{Al}_{0.025} \mathrm{Sn}_{0.025} \mathrm{O}_{3}$. However, all the $\beta$ values obtained are smaller than 0.5 , indicating the existence of magnetic inhomogeneities and/or AFM phase on the surface layers of our sample.

In order to manifest the influence of critical exponents on MCE, the field dependence of the entropy change was also analyzed. According to Oesterreicher et al., for a material exhibiting a second order phase transition, the field dependence of the peak magnetic entropy change $\Delta S_{\mathrm{M}}^{\mathrm{pk}}$ can be expressed as: ${ }^{46}$

$$
\left|\Delta S_{\mathrm{M}}^{\mathrm{k}}\right|=a(H)^{n}
$$

where the exponent $n$ is a local exponent related to magnetic order and depends on temperature and magnetic field. It can be locally calculated as follows:

$$
n=\frac{\mathrm{d} \ln \Delta S_{\mathrm{M}}}{\mathrm{d} \ln \left(\mu_{0} H\right)}
$$

Furthermore, at $T=T_{\mathrm{C}}$, the exponent $n$ becomes field independent and is given by: ${ }^{47}$

$$
n\left(T_{\mathrm{C}}\right)=1+\frac{\beta-1}{\beta+\gamma}
$$

where $\beta, \gamma$ and $\delta$ are the critical exponents. Through the Widom relation $(\delta=1+\gamma / \beta)$, eqn (12) becomes:

$$
n=1+\frac{1}{\delta}\left(1-\frac{1}{\beta}\right)
$$

Using the order parameters $\beta, \gamma$ and $\delta$ at $T=300 \mathrm{~K}$, obtained from the KF method, the value of $n$ calculated from the above relation is found to be 0.61 . This value is in good agreement with the predicted value of $2 / 3$ in the mean field approach. ${ }^{48}$ In order to check the validity of $n$ value, the experimental data of field dependence of $\Delta S_{\mathrm{M}}$ at transition temperature for the $\mathrm{La}_{0.7} \mathrm{Ca}_{0.1} \mathrm{~Pb}_{0.2} \mathrm{Mn}_{0.95} \mathrm{Al}_{0.025} \mathrm{Sn}_{0.025} \mathrm{O}_{3}$ sample is plotted in Fig. 10. The fitting of full square points $\left(\Delta S_{\mathrm{M}}^{\max }\right)$, according to eqn (11), gives $n\left(T_{\mathrm{C}}\right)=0.7$, which is higher than those calculated from eqn (13). The deviation from the mean field behavior is due to the presence of local inhomogeneities around the Curie temperature.

On the other hand, the field dependence of the relative cooling power (RCP) for $\mathrm{La}_{0.7} \mathrm{Ca}_{0.1} \mathrm{~Pb}_{0.2} \mathrm{Mn}_{0.95} \mathrm{Al}_{0.025} \mathrm{Sn}_{0.025} \mathrm{O}_{3}$ was also analyzed and shown in Fig. 10. The RCP, defined as the product of the peak entropy change times the full width at half maximum, should scale with field as a power law, with an exponent $1+1 / \delta$. The field dependence of the RCP is analyzed using the following relation:

$$
\mathrm{RCP} \propto H^{1+1 / \delta}
$$

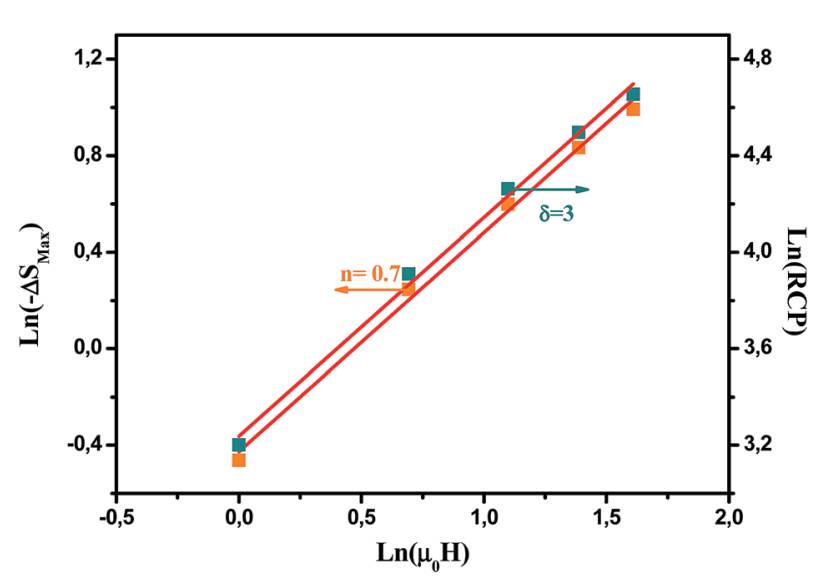

Fig. 10 Field dependence of the $-\Delta S_{M}^{\max }$ and the RCP calculated from the magnetic data, with the calculated $n$ and $\delta$ for both slopes. 
where $\delta$ is the critical exponent of the magnetic transition. The value $\delta$ calculated from the slope is 3, which is in agreement with those obtained using the critical isotherms analysis (CIA), modified Arrott plots (MAP) and Kouvel-Fisher method (KF). This confirms the good correlation between critical behavior and magnetocaloric effect.

Franco and co-workers used the scaled equation of state ${ }^{31,49}$ and the critical exponents, which is written as $\frac{\mu_{0} H}{M^{\delta}}=f\left[\frac{\varepsilon}{M^{1 / \beta}}\right]$, to scaling $\Delta S$ in the critical region (around $T_{\mathrm{C}}$ ). By using the scaled magnetic equation of state and eqn (1), Franco et al. were capable of showing that: ${ }^{50}$

$$
-\Delta S\left(\mu_{0} H, T\right)=\left(\mu_{0} H\right)^{(1-\alpha) / \Delta} g\left(\frac{\varepsilon}{\left(\mu_{0} H\right)^{1 / \Delta}}\right)
$$

where $\alpha$ and $\Delta$ are the usual critical exponents. We calculated $\Delta$ and $\alpha$ using the relations ${ }^{51} \Delta=\beta+\gamma$ and $\alpha+2 \beta+\gamma=2$. Thus, according to $\Delta S^{\prime}=\left(T, \Delta H=H_{\max }\right)=\frac{\Delta S\left(T, \Delta H=H_{\max }\right)}{\Delta S^{\text {peak }}\left(T, \Delta H=H_{\max }\right)}$ (ref. 15) and using the appropriate values for the critical exponents and the Curie temperature, Franco et al. plotted their experimental data as $\frac{-\Delta S\left(\mu_{0} H, T\right)}{\left(\mu_{0} H\right)^{1-\alpha / \Delta}}$ vs. $\frac{\varepsilon}{\left(\mu_{0} H\right)^{1 / \Delta}}$ and found that all the data for all temperatures and fields collapse on a single curve, which proves the validity of eqn (18). Using the values of $\beta, \gamma$, and $T_{\mathrm{C}}$ obtained from the modified Arrott plots method, the scaled data are plotted in Fig. 11. All data for our sample collapse on a single master curve for all measured fields and temperatures. The excellent overlap of the experimental data points clearly indicates that the obtained values of $\beta, \gamma$, and $T_{\mathrm{C}}$ for this compound is in agreement with the scaling hypothesis near magnetic transition.

In addition, in homogeneous magnets the university class of the magnetic phase transition deduced from the normalization group analysis depends strongly on the range of the exchange interaction as it is described by this equation:

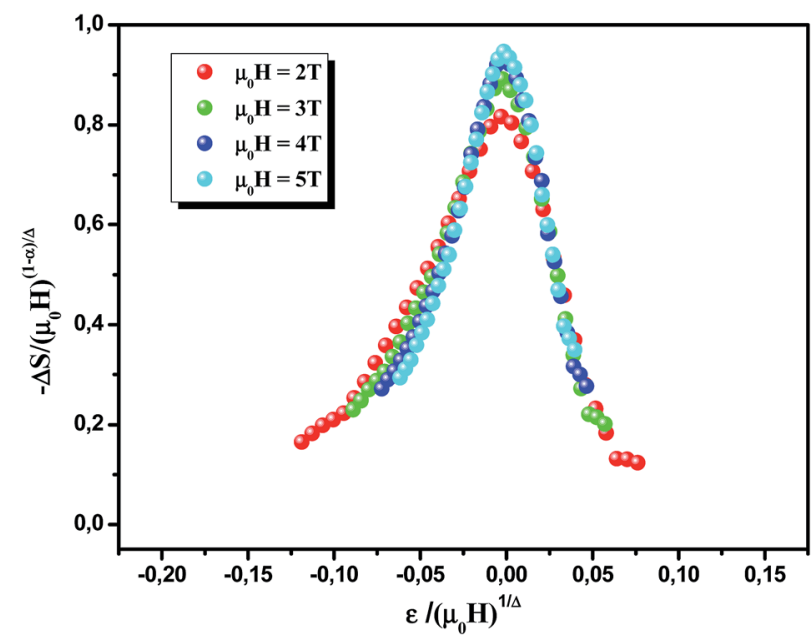

Fig. 11 Scaled magnetic entropy change vs. scaled temperature using critical exponents.

$$
j(r)=\frac{1}{r^{d+\sigma}}
$$

where $d$ is the space dimensionality, $\sigma$ is the range of interaction and $r$ determines the range of exchange interaction. ${ }^{52}$ For a three-dimensional material $(d=3)$, the relationship is $j(r)=$ $1 / r^{3+\sigma}$ with $3 / 2 \leq \sigma \leq 2$. Generally, the MF-theory $(\beta=0.5, \gamma=$ 1.0 and $\delta=3.0$ ) exponents are valid for $\sigma<1 / 2$, which indicates $j(r)$ decreases with "long-range" distance slower than $r^{-4.5}$, while the Heisenberg ones are for $\sigma>2$. In the intermediate range, if $1 / 2<\sigma<2$, the exponents belong to other universality classes (such as the TMF theory and 3D-Ising model). In the case of our samples, the values of the critical exponents are in agreement with the mean-field model. Consequently $(d+\sigma)$ will be smaller than 4.5 , and the exchange interaction $j(r)$ drops slower than $r^{-4.5}$ for our sample.

Moreover, the influence of disorder on the critical behavior of magnetic systems whose pure version undergoes a second order phase transition is predicted by the criterion of Harris. ${ }^{53}$ In this context, Harris asserts that if the critical exponent $\alpha_{\text {pure }}$ of the pure system is positive, the disorder changes the critical exponents. While, if $\alpha_{\text {pure }}$ is negative, the disorder is irrelevant. The $\alpha_{\text {pure }}$ exponent for the pure system is calculated using the Rushbrooke scaling relation given by:

$$
\alpha_{\text {pure }}+2 \beta+\gamma=2
$$

Based on this relation, the exponent $\alpha_{\text {pure }}$ is found to be positive which implies that the disorder is relevant.

In the following section, we will use the obtained magnetic entropy change to study the spontaneous magnetization $(M)$ in our material. A general result issued from a mean-field theory reveals the dependence of the magnetic entropy on the relative magnetization can be described as: ${ }^{54-57}$

$$
\begin{aligned}
& S(\sigma)= \\
& -N k_{\mathrm{B}}\left[\ln (2 J+1)-\ln \left(\frac{\sinh \left(\frac{2 J+1}{2 J} B_{\mathrm{J}}^{-1}(\sigma)\right)}{\sinh \left(\frac{1}{2 J} B_{\mathrm{J}}^{-1}(\sigma)\right)}\right)+B_{\mathrm{J}}^{-1}(\sigma) \times \sigma\right]
\end{aligned}
$$

where $\sigma=M / g \mu \beta J N, M$ is the magnetization, $N$ is the number of spins, $J$ is the spin value, $k_{\mathrm{B}}$ and $B_{\mathrm{J}}$ are the Boltzmann constant and the Brillouin function respectively.

From a power expansion of eqn (17), $\Delta S_{\mathrm{M}}$ is proportional to $M^{2}$ and, from the mean-field model, for small $M$ values: ${ }^{48,56}$

$$
-S(\sigma)=\frac{3 J}{2 J+1} N k_{\mathrm{B}} \sigma^{2}+0\left(\sigma^{4}\right)
$$

However, in the ferromagnetic state (below $T_{\mathrm{C}}$ ) the sample indicates a spontaneous magnetization and the $\sigma=0$ state is never attained. Explicitly, and considering only the first term of the expansion of eqn (18), the magnetic entropy change may be written as: ${ }^{58}$ 


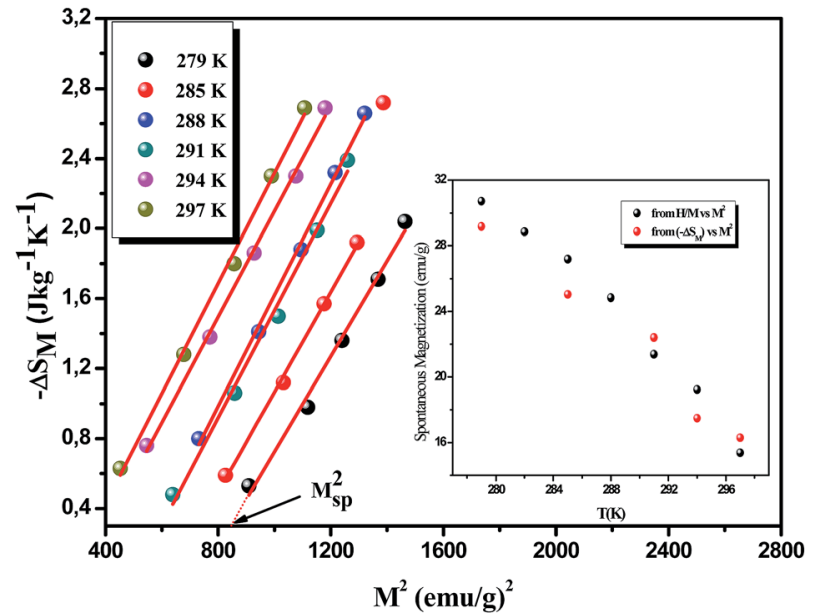

Fig. 12 Isothermal $-\Delta S_{M}$ vs. $M^{2}$ curves (the solid lines are linear fits to the data). The inset: spontaneous magnetization of $\mathrm{La}_{0.7} \mathrm{Ca}_{0.1} \mathrm{~Pb}_{0.2^{-}}$ $\mathrm{Mn}_{0.95} \mathrm{Al}_{0.025} \mathrm{Sn}_{0.025} \mathrm{O}_{3}$ deduced from the extrapolation of the isothermal $-\Delta S_{M}$ vs. $M^{2}$ curves and from the Arrott plots $\mu_{0} H / M$ vs. $M^{2}$.

$$
-S(\sigma)=\frac{3 J}{2 J+1} N k_{\mathrm{B}}\left(\sigma^{2}-\sigma_{\text {spon }}^{2}\right)
$$

The mathematical analysis of this equation shows that the isotherms of the variation of the magnetic entropy $(-\Delta S)$ with the square of the magnetization $\left(M^{2}\right)$ in the ferromagnetic region (FM) have a linear variation. The curves constructed in Fig. 12 in the temperature range between $279 \mathrm{~K}$ and $297 \mathrm{~K}$ confirms clearly the former suggestion with an approximately constant slope throughout the FM region. Moreover, which results in a shift of the isothermal $\Delta S_{\mathrm{M}}$ vs. $M^{2}$ plots in the ferromagnetic region, with an horizontal drift from the origin corresponding to the value of $M_{\text {spont }}{ }^{2}(T)$, whereas for $T>T_{\mathrm{C}}$ the $\Delta S_{\mathrm{M}}$ vs. $M^{2}$ plots start at a null $M$ value. The spontaneous magnetization $(M(T))$ is then estimated, and compared to the results obtained from the Arrott curves, as shown in the inset of Fig. 12.

Using eqn (4) and after many repetitions of the analysis fits, the critical exponent $\beta$ determined by the Landau model is found to be $0.52 \pm 0.41$. The good agreement between the two methods allows to prove the validity of this recipe to estimate the spontaneous magnetization using a mean-field analysis of the magnetic entropy change in our sample.

\section{Conclusion}

To summarize, we have investigated the critical behavior and spontaneous magnetization in $\mathrm{La}_{0.7} \mathrm{Ca}_{0.1} \mathrm{~Pb}_{0.2} \mathrm{Mn}_{0.95} \mathrm{Al}_{0.025^{-}}$ $\mathrm{Sn}_{0.025} \mathrm{O}_{3}$ sample. The reliable exponents $\left(T_{\mathrm{C}}, \beta, \gamma\right.$ and $\left.\delta\right)$ are obtained based on various research techniques including modified Arrott plot, Kouvel-Fisher method, and critical isotherm analysis. It is found that the estimated critical exponents confirm that the experimental data agree well with the theoretical prediction of the mean-field model, and are dominated by a long range interactions around $T_{\mathrm{C}}$. Moreover, a perfect agreement was gained between two different techniques for determining the spontaneous magnetization by modeling the magnetic entropy change of second order magnetic phase transitions.

\section{Conflicts of interest}

There are no conflicts to declare.

\section{References}

1 M. H. Phan and S. C. Yu, J. Magn. Magn. Mater., 2007, 308, 325.

2 A. Kitanovski and P. W. Egolf, J. Magn. Magn. Mater., 2009, 321, 777.

3 J. B. Goodenough, J. Appl. Phys., 1997, 81, 5330.

4 A. J. Millis, B. I. Shraiman and R. Mueller, Phys. Rev. Lett., 1996, 77, 175.

5 C. Zener, Phys. Rev., 1951, 82, 403.

6 Y. Motome and N. Furulawa, J. Phys. Soc. Jpn., 2000, 69, 3785.

7 Y. Motome and N. Furulawa, J. Phys. Soc. Jpn., 2001, 70, 1487.

8 K. Ghosh, C. J. Lobb, R. L. Greene, S. G. Karabashev, D. A. Shulyatev, A. A. Arsenov and Y. Mukovskii, Phys. Rev. Lett., 1998, 81, 4740.

9 C. V. Mohan, M. Seeger, H. Kronmüller, P. Murugaraj and J. Maier, J. Magn. Magn. Mater., 1998, 183, 348.

10 A. Omri, A. Tozri, M. Bejar, E. Dhahri and E. K. Hlil, J. Magn. Magn. Mater., 2012, 324, 3122.

11 D. Ginting, D. Nanto, Y. D. Zhang, S. C. Yu and T. L. Phan, Phys. B, 2013, 421, 17.

12 L. Chen, J. H. He, Y. Mei, Y. Z. Cao, W. W. Xi, H. F. Xu, Z. W. Zhu and Z. A. Xu, Phys. B, 2009, 404, 1879-1882.

13 J. Fan, L. Ling, B. Hong, L. Zhang, L. Pi and Y. Zhang, Phys. Rev. B: Condens. Matter Mater. Phys., 2010, 81, 144426.

14 K. Dhahri, N. Dhahri, J. Dhahri, K. Taibi, E. K. Hlil, H. Belmabrouk and M. Zaidi, RSC Adv., 2017, 7, 4341043423.

15 K. Dhahri, N. Dhahri, J. Dhahri, K. Taibi and E. K. Hlil, J. Alloys Compd., 2017, 699, 619-626.

16 C. Vazquez-Vazquez, M. C. Blanco, M. A. Lopez-Quintela, R. D. Sanchez, J. Rivas and S. B. Oseroff, J. Mater. Chem., 1998, 8(4), 991.

17 H. L. Ju and H. Sohn, J. Magn. Magn. Mater, 1997, 167, 200. 18 K. A. Gschneidner, V. K. Pecharsky and A. O. Tsokol, Rep. Prog. Phys., 2005, 68, 1479.

19 D. T. Morelli, A. M. Mance, J. V. Mantese and A. L. Micheli, J. Appl. Phys., 1996, 79, 373.

20 V. K. Pecharsky and K. A. Gschneidner Jr, Phys. Rev. Lett., 1997, 78, 4494.

21 D. N. H. Nam, N. V. Dai, L. V. Hong, N. X. Phuc, S. C. Yu, M. Tachibana and E. Takayama-Muromachi, J. Appl. Phys., 2008, 103, 043905.

22 Y. Sun, M. B. Salamon and S. H. Chun, J. Appl. Phys., 2002, 92, 3235.

23 X. Bohigas, J. Tejada, E. Del Barco, X. X. Zhang and M. Sales, Appl. Phys. Lett., 1998, 73, 390. 
24 N. Chau, H. N. Nhat, N. H. Luong, D. L. Minh, N. D. Tho and N. N. Chau, Phys. B, 2003, 327, 270.

25 B. K. Banerjee, Phys. Lett., 1964, 12, 16.

26 J. Mira, J. Rivas, F. Rivadulla, C. Vazquez and M. A. LopezQuintela, Phys. Rev. B: Condens. Matter Mater. Phys., 1999, 60, 2998-3001.

27 H. E. Stanley, Introduction to Phase Transitions and Critical Phenomena, Oxford University Press, London, 1971.

28 A. Arrott and J. E. Noakes, Phys. Rev. Lett., 1967, 19, 786.

29 H. E. Stanley, Rev. Mod. Phys., 1999, 71, S358.

30 H. S. Shin, J. E. Lee, Y. S. Nam, H. L. Ju and C. W. Park, Solid State Commun., 2001, 118, 377.

31 B. Widom, J. Chem. Phys., 1965, 43, 3898.

32 C. V. Mohan, M. Seeger, H. Kronmüller, P. Murugaraj and J. Maier, J. Magn. Magn. Mater., 1998, 183, 348.

33 J. S. Kouvel and M. E. Fisher, Phys. Rev., 1964, 136, A1626.

34 B. Yu, W. Sun, J. Fan, X. Lan, W. Zhang, Y. Zhu, H. Han, L. Zhang, L. Ling and H. Yang, Mater. Res. Bull., 2018, 99, 393-397.

35 N. Dhahri, J. Dhahri, E. K. Hlil and E. Dhahri, J. Magn. Magn. Mater., 2012, 324, 806-811.

36 L. Xu, J. Fan, W. Sun, Y. Zhu, D. Hu, J. Liu, Y. Ji, D. Shi and J. Hao Yang, Appl. Phys. Lett., 2017, 111(5), 052406.

37 M. Baazaoui, S. Hcini, M. Boudard, S. Zemni and M. Oumezzine, J. Magn. Magn. Mater., 2016, 401, 323-332.

38 M. H. Phan, V. Franco, N. S. Bingham, H. Srikanth, N. H. Hur and S. C. Yu, J. Alloys Compd., 2010, 508, 238.

39 J. Yang and Y. P. Lee, Appl. Phys. Lett., 2007, 91, 142512.

40 N. Ghosh, S. Rößler, U. K. Rößler, K. Nenkov, S. Elizabeth, H. L. Bhat, K. Dörr and K. H. Müller, J. Phys.: Condens. Matter, 2006, 18, 557.

41 D. Ginting, D. Nanto, Y. D. Zhang, S. C. Yu and T. L. Phan, Phys. B, 2013, 412, 17.
42 M. Ziese, J. Phys.: Condens. Matter, 2001, 13, 2919.

43 S. N. Kaul, J. Magn. Magn. Mater., 1985, 53, 5.

44 K. Huang, Statistical Mechanics, Wiley, New York, 2nd edn, 1987.

45 J. L. Alonso, L. A. Fernández, F. Guinea, V. Laliena and V. Martín-Mayor, Nucl. Phys. B, 2001, 596, 587.

$46 \mathrm{H}$. Oesterreicher and F. T. Parker, Magnetic cooling near Curie temperatures above $300 \mathrm{~K}$, J. Appl. Phys., 1984, 55, 4334.

47 J. Fan, L. Pi, L. Zhang, W. Tong, L. Ling, B. Hong, Y. Shi, W. Zhang, Di Lu and Y. Zhang, Appl. Phys. Lett., 2011, 98(7), 072508.

48 M. Pekala, J. Appl. Phys., 2010, 108, 113913.

49 R. B. Griffiths, Phys. Rev., 1967, 158, 176.

50 V. Franco, J. S. Blázquez and A. Conde, J. Appl. Phys., 2008, 103, $07 \mathrm{~B} 316$.

51 A. Hankey and H. E. Stanley, Phys. Rev. B: Solid State, 1972, 6, 3515.

52 A. K. Pramanik and A. Banerjee, Phys. Rev. B: Condens. Matter Mater. Phys., 2009, 79, 214426.

53 A. B. Harris, J. Phys. C: Solid State Phys., 1974, 7, 1671.

54 K. Huang, Statistical Mechanics, Wiley, New York, 2nd edn, 1987.

55 J. S. Amaral and V. S. Amaral, J. Magn. Magn. Mater., 2010, 322, 1552.

56 G. J. Liu, J. R. Sun, J. Lin, Y. W. Xie, T. Y. Zhao, H. W. Zhang and B. G. Shen, Appl. Phys. Lett., 2006, 88(21), 212505.

57 L. Xu, J. Fan, Y. Zhu, Y. Shi, L. Zhang, L. Pi, Y. Zhang and D. Shi, Mater. Res. Bull., 2016, 73, 187-191.

58 X. Zhang, J. Fann, L. Xu, D. Hu, W. Zhang and Y. Zhu, Ceram. Int., 2016, 42(1), 1476-1481. 